\title{
Geometrical precision alignment of the Micromegas detectors for the ATLAS New Small Wheel upgrade
}

\author{
Manisha Lohan \\ (on behalf of ATLAS Muon Collaboration) \\ IRFU, CEA, Université Paris-Saclay, Gif-sur-Yvette 91191, France \\ E-mail: manisha.lohan@cern.ch
}

\begin{abstract}
The upgrade of the Large Hadron Collider to the High Luminosity LHC is required to probe the physics beyond Standard Model. After the ongoing long shutdown and eventually after LS3 in 2026, the accelerator luminosity will be increased up to 7 times as compared to design luminosity $\left(10^{34} \mathrm{~cm}^{-2} \mathrm{~s}^{-1}\right)$. To meet the requirements of higher rates environment of HL-LHC era, the muon system of ATLAS detector needs to be upgraded. Therefore, the present small wheel will be replaced by the New Small Wheel, which will be constituted by MicroMegas micro-pattern gaseous detectors and small-strip Thin Gap Chambers. The methods adopted to achieve the challenging required geometrical precision of Micromegas chambers are presented. Specific measurement devices have been developed in the last few years to determine the mechanical metrology quality of Micromegas chambers, required for NSW. Planarity measurements of panels as well as modules after assembly are done with a specifically developed co-ordinate measuring machine. The methodology and the results will be presented. Results of in-plane measurements performed using Rasnik masks etched on the PCBs are also reported.
\end{abstract}

ICHEP2020, 40th International Conference on High Energy Physics

28 July - 6 August, 2020

Virtual Conference 


\section{Introduction}

The ATLAS experiment [1] requires an upgrade to benefit from the High Luminosity LHC (HL-LHC) [2] era. The New Small Wheel (NSW) project [3] is one of the largest upgrade projects of the ongoing long shutdown (LS2). The NSW has two detection technologies: Micromegas (MM) for precision tracking and small-strip Thin Gap Chambers for triggering purpose (even though each technology could do both - tracking and triggering). For each of the two NSWs, 16 modules will be installed in 16 sectors i.e. 8 large sectors and 8 small sectors; covering total area of $\sim 1200$ $\mathrm{m}^{2}$. Each sector is split in two sections radially so that there are four types of MM unit detectors referred as SM1, SM2, LM1, and LM2 types, covering between 2 and $3 \mathrm{~m}^{2}$. Each of these MM modules contains 4 detection gaps obtained by assembling 5 composite panels.

LM1 panels construction is a few steps process, performed sequentially on a granite table [4] equipped with $8 \mathrm{~cm}$ thick granite assembly table. The granite assembly table has metallic pins at specific positions for alignment of panel corners and each of the printed circuit board (PCB). First of all, 5 PCBs are assembled to form the first side of a panel. The flatness of the granite sole guarantees the flatness of this side. The PCBs are precisely positioned on the granite sole using the precision washers glued precisely w.r.t. a reference strip on each PCB. These precision washers match the precision pins of the granite sole. Then honeycomb pieces and aluminum bars are glued on the PCBs and after an overnight cure of the glue, the previously glued half panel is removed and stored on a flat Stiff-back. Finally a layer of 5 PCBs forming the second side of the panel is prepared in a similar way and glued with the half panel. V-shape and L-shape used to align panels during module assembly are glued during the second step of panel preparation. Each granite table is equipped with a movable 3-axes gantry allowing precise positioning of $\sim 10 \mu \mathrm{m}$ on both axes in the horizontal plane of both a camera, used for alignment controls, and an optical sensor dedicated to measure the third coordinate of panels with a precision of $\sim 10 \mu \mathrm{m}$, or to perform a final detector planarity control. This latter functionality is extensively used at each step of the panel construction up to its completion as panel flatness directly affects the Micronmegas module tracking precision. More details about planarity and positioning measurements of panels as well as modules are described in next sections.

\section{Planarity measurements of panels and modules}

The thickness of a panel is measured at a few millions points on its surface using an optical sensor tool. This scan allows to check that the mean panel thickness, the thickness RMS and extremal thicknesses are within prescribed ranges as well as to spot localized bump or dip areas. As shown in Figure 1 left, except for a few cases, there are no deviations outside acceptable range of $11.4 \mathrm{~mm} \pm 0.110 \mathrm{~mm}$ for drift and $11.8 \mathrm{~mm} \pm 0.110 \mathrm{~mm}$ for readout panel. The equivalent measurements are done on the modules as shown in Figure 1 right.

\section{Measurements of PCB positions within panels and modules}

Alignment measurements of two faces of a readout panel are performed with Rasnik masks [5] etched on every PCB and a specific device named Rasfork. The Rasfork is composed of two tubes, 

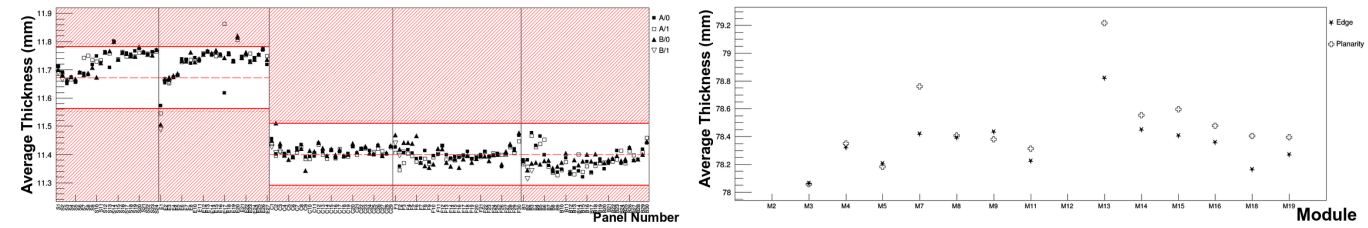

Figure 1: Average thickness of panels vs. panel number (left). Average thickness of modules vs. module number (right).

each equipped with a prism, lens and CCD, such that when inserted on a panel edge, the Rasnik masks on the two faces are imaged on the CCDs. To do the measurement, the panel is placed on $25 \mathrm{~mm}$ shims and Rasnik images are recorded. The measurement is repeated for each of the 30 mask pairs of a readout panel. By comparing the two images, the extent of the mis-alignment of one face w.r.t. the other is calculated. Analyzing the collected data, PCB placement on the panel is reconstructed as shown in Figure 2 left. In general, the mis-alignment is better than $30 \mu \mathrm{m}$ and $30 \mu \mathrm{rad}$. Alignment measurements of modules is done in similar way using 4-channel Rasfork in order to measure both readout panels at the same time. In general, two panels of a module have mis-alignment better than $50 \mu \mathrm{m}$ and $50 \mu \mathrm{rad}$ as shown in Figure 2 right.
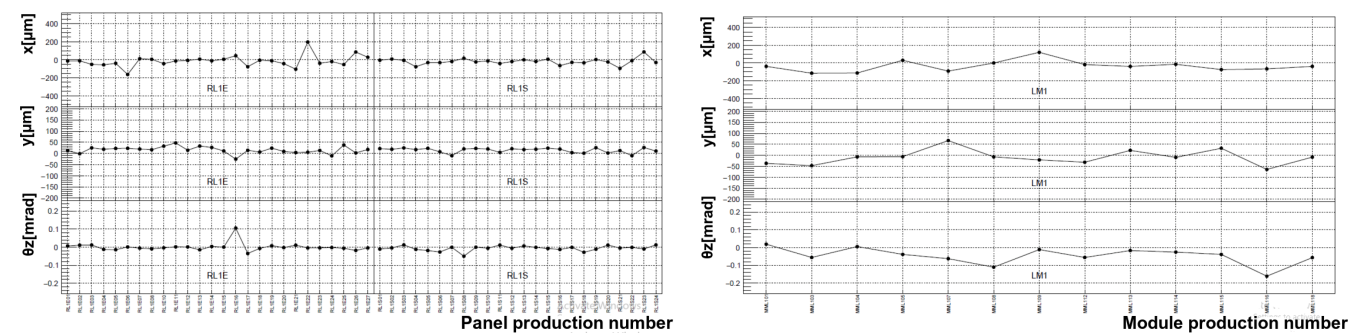

Figure 2: Translational and rotational deformation parameters of panel layer B w.r.t. layer A (left plot), translational and rotational deformation parameters of stereo panel w.r.t. eta panel (right plot).

\section{Conclusion}

The required and challenging specifications for planarity of panels and modules are achieved consistently in the measurements of various panels and modules. Alignment measurements for panels and modules produced consistent results, also within acceptable range.

\section{References}

[1] ATLAS Collaboration, The ATLAS experiment at the CERN Large Hadron Collider, JINST 3 (2008) S08003.

[2] G. Apollinari et al., High-luminosity large hadron collider (hl-lhc) preliminary design report, CERN-2015-005.

[3] T. Kawamoto et al., New Small Wheel Technical Design Report, ATLAS-TDR-20-2013.

[4] Microplan SAS France, https://www.microplan-group.com/fr/

[5] M. Beker et al., The Rasnik 3-point optical alignment system, 2019 JINST 14 P08010. 